\title{
RETICULARIDAD E INNOVACIÓN EN ACTORES Y STAKEHOLDERS DE TURISMO DE SALUD: CASO DE COMPETITIVIDAD EN LOS ALGODONES, BAJA CALIFORNIA
}

\author{
Reticularity and innovation in actors and \\ stakeholders of health tourism: competitiveness \\ case in Los Algodones, Baja California
} noma de Ciudad Juárez. Correo electrónico: izizaldr@uacj.mx. (D) ORCID: http://orcid.org/0000-0001-7079-5521 


\section{Resumen}

El manuscrito explora la reticularidad y la innovación en el ámbito del turismo. Estudios recientes en procesos de competitividad, sugieren que las organizaciones interesadas se constituyan en redes, las cuales alienten la cooperación con el propósito de hacerse fuertes y alcanzar metas en conjunto. Bajo este contexto, el objetivo de esta investigación consiste en analizar la reticularidad de actores y stakeholders, que a través de la colaboración entre ellos permite la innovación en la creación de nuevos productos y servicios, más eficientes procesos y sistemas de trabajo, mejora en los métodos de organización, así como a la introducción de novedosas formas de mercadeo; todo lo anterior, dirigido al turismo de salud en la región de influencia de Los Algodones, B. C. (LAL). La investigación consistió en un estudio cuanticualitativo, mediante la metodología de Análisis de Redes Sociales (ARS), aplicando entrevistas semiestructuradas a actores líderes de la actividad de turismo de salud. Los resultados obtenidos ponen en evidencia que las redes entre actores y stakeholders se encuentran afianzadas en relaciones de confianza, respeto y compromiso al desarrollo de la actividad turística; estas permiten procesos de colaboración que generan innovación e influyen en capacitación del recurso humano y estrategias de promoción conjunta.

Palabras clave: colaboración, redes, análisis de redes sociales, innovación, turismo de salud.

\section{Abstract}

The manuscript explores reticularity and innovation in the field of tourism. Recent studies in competitiveness processes suggest that interested organizations become networks, which encourage cooperation with the purpose of becoming strong and reaching goals together. In this context, the objective of this research is to analyze the reticulation of actors and stakeholders, which through collaboration between them allows innovation in the creation of new products and services, more efficient processes and systems of work, improvement in methods of organization, as well as the introduction of novel forms of marketing; all of the above, aimed at health tourism in the region of influence of Los Algodones, B. C. (LAL). The research consisted of a quantum-qualitative study, using the methodology of Social Network Analysis (ARS), applying semi-structured interviews to leading actors in the tourism activity of health. The results obtained show that the networks between actors and stakeholders are established in relationships of trust, respect and commitment to the development of tourism activity; These allow collaborative processes that generate innovation and influence human resource training and joint promotion strategies.

Keywords: collaboration, networks, social network analysis, innovation, health tourism. 


\section{Introducción}

$\mathrm{E}$ l estudio de la actividad de turismo no puede concebirse como el trabajo aislado de operadores económicos; al contrario, se enfoca como un conjunto de interesados en franca relación, compartiendo objetivos, recursos tecnológicos, informáticos y humanos. En este contexto, surgen redes de colaboración que según Verschoore y Balestrin (2008) se interpretan como un conjunto de organizaciones con objetivos comunes, formalmente relacionadas, con un plazo ilimitado de existencia y en la que cada miembro mantiene su competencia individual.

En este sentido, la actividad turística mantiene altos grados de interdependencia donde la evolución o creación de relaciones de colaboración, tales como redes, es más que un resultado natural; es un imperativo gerencial. Se refiere a un acto voluntario de los diferentes actores involucrados en la actividad que busca alcanzar, a través de la unión de esfuerzos, beneficios y objetivos que serían difíciles de lograr por si solos. Por lo tanto, la cooperación es una prioridad en la coordinación de esfuerzos regionales, donde sin duda, la dependencia de una gestión exitosa del turismo requiere de un enfoque mucho más colaborativo por parte de las comunidades locales, pequeñas empresas y operadores individuales.

Bajo este esquema, los actores turísticos de un territorio y sus relaciones de colaboración y coordinación son un elemento necesario para la articulación efectiva de un destino turístico y su actividad prioritaria, ya que cobran especial atención las estrategias y acciones que emprenden para adaptarse a las condiciones del contexto. Por tanto, se considera que para el desarrollo de la innovación en un destino turístico debe estar favorecida por el intercambio de ideas y proyectos entre una red de actores que lo dinamicen (Hjalager, 2010); en otras palabras, en la medida que hay colaboración mejoran las posibilidades de innovación.

Es decir, la dinámica de innovación es producto de las relaciones de colaboración entre actores y stakeholders que interactúan para desarrollar actividades en el proceso productivo de un determinado territorio. A este respecto, Merinero y Pulido (2016), pone de relieve la colaboración y las relaciones entre los diversos actores (públicos o privados) como elemento clave para comprender el desarrollo de los procesos innovadores y por ende, de la competitividad empresarial.

\section{Planteamiento del problema}

El modelo de competitividad a través de la colaboración también se despliega en los servicios internacionales de salud, que se encuentran entre las actividades de mayor crecimiento a nivel mundial, estimado en tres billones de dólares anuales en promedio (Vargas-Hernández, 2011). Situación favorecida por la búsqueda de la salud a mejores costos, mayor calidad, minimización de listas de espera, entre otras referencias que han permitido a diferentes destinos en el mundo posicionarse en esta actividad económica; definida como un amplio conglomerado de organizaciones de salud, turísticas, gubernamentales, académicas y de investigación.

En este contexto, han aparecido un conjunto de publicaciones que refieren la temática de innovación y competitividad en los servicios turísticos, tales como Decelle (2004), Vilaseca et al. (2007), González 
y Mendieta. (2009), Antón y Duro (2009), Hjalager (2010), Carlise et al. (2013), Ivars et al. (2014). Sin embargo, se observa una laguna en la investigación desde el enfoque de Análisis de Redes Sociales (ARS) para la innovación en turismo de salud, por lo que el trabajo que ahora se presenta, intenta subsanar algunas de esas carencias, dado los nuevos patrones de consumo y de producción de servicios de salud en las últimas décadas, implicando el desplazamiento de los pacientes a través de las fronteras en la búsqueda de servicios médicos.

Bajo esta expectativa, el comercio internacional de servicios médicos en los países en desarrollo ha aparecido en la escena como una forma de incrementar sus ingresos y en el caso de México se ha convertido en un segmento estratégico participando con un 2.46\% del mercado de exportación de servicios de salud a nivel mundial, aspecto que representa la afluencia de más de un millón de visitantes anuales. En este entorno cada vez más competitivo, la principal preocupación es atraer a nuevos turistas de la salud a través de la comercialización de un paquete de bienes y servicios en donde los beneficios obtenidos sean compartidos entre la red de actores que forman parte de la actividad económica del destino.

En consecuencia, los diferentes grupos de interesados observan el beneficio de innovar en acciones conjuntas de desarrollo, dado que los viajes médicos y de salud, en su mayor parte, se llevan a cabo en el sector privado sin la regulación y la remisión formal por proveedores de salud pública. De esta forma, los vínculos informales en la estructura social entre los actores de turismo de salud cobran especial importancia, dado que, como señala (Hjalager, 2010), los actores son agentes humanos cuyas decisiones se ven afectadas por sus amigos, por los amigos de sus amigos y potencialmente incluso, por aquellos que están lejos de lo que se denomina su horizonte de observabilidad.

En estos términos, es procedente preguntarse: i) ¿cómo se organizan los actores y stakeholders de turismo de salud en Los Algodones?; ii) ¿existe colaboración entre ellos?; y, si dichos procesos iii) ¿generan innovación en esta actividad económica? Por consiguiente, el presente trabajo de investigación, analiza la estructura reticular de actores y stakeholders en el turismo de salud; en el entendido que la colaboración se intensifica por las relaciones de respeto y compromiso al desarrollo de acciones de colaboración para la generación de innovación en el destino de Los Algodones, B. C., bajo el esquema del modelo de ARS, considerado un vasto y consolidado campo de trabajo en las áreas de turismo.

\section{Revisión de literatura}

No puede negarse que vivimos en mundo de interconexiones. En este entorno, la creciente importancia de la innovación y la adaptación a modelos más competitivos ha cambiado la naturaleza de las relaciones entre las organizaciones, por lo que, con mayor frecuencia, aparecen estructuras reticulares como alianzas, asociaciones o comunidades empresariales. En estos términos, la actividad turística es un amplio aglutinamiento de organizaciones, que en mayor o menor medida participan e incentivan el desarrollo de la actividad, verbigracia organizaciones gubernamentales, agrupaciones privadas, centros educativos, organismos empresariales, visitantes, proveedores, promotores y sobre todo la comunidad del destino (Molina, Cuevas y Rodríguez, 2014). Estos colectivos a los que se hace referencia son conocidos como stakeholders, es decir, los que aportan recursos a la organización y tienen interés en su desarrollo (Freeman, Wicks y Parmar, 2004). 
Así, el turismo, es susceptible investigarse bajo el enfoque de Análisis de Redes Sociales (ARS), que como señala Scott (2017), los apegos individuales pasan a segundo término para priorizar los sistemas relacionales de los agentes construidos a partir de las partes conectadas de agentes interactivos. Por lo tanto, el ARS proporciona un medio para promover la colaboración efectiva dentro de los destinos. Permite la identificación de momentos críticos que cruzan las fronteras individualistas y competitivas, permitiendo la integración de los grupos interesados a partir de iniciativas estratégicas de innovación para la competitividad de los destinos. El presente trabajo estudia las relaciones de colaboración para la innovación bajo en ARS.

\subsection{Teoría de los stakeholders y de vínculos}

Un grupo, se califica como interesado si tiene un interés legítimo en los aspectos de la organización y por tanto, posee el poder de afectar a la empresa, sus rendimientos o tiene una participación en la misma (Sautter y Leisen, 2009). Bajo esta perspectiva, es conveniente el desarrollo de relaciones de confianza y cooperación mutua con sus stakeholders, creando comunidades en las que cada parte se esfuerce en generar valor para el sistema en su conjunto. La gestión de los vínculos que la organización establece con sus stakeholders contribuye a la generación de innovación, y como consecuencia, de ventajas competitivas.

Consecuentemente, resulta importante la identificación de los stakeholders a partir de los vínculos que establecen con el sistema. Gruning y Hunt (1984), modelaron la Teoría de Vínculos que sostienen que los sistemas empresariales están relacionados con sus stakeholders por los recursos aportados y arriesgados para su supervivencia (Baro, 2011). Debido a lo anterior, se expresan cuatro tipos de vínculos: a) los posibilitadores, que proporcionan la infraestructura, el marco regulatorio y la financiación (por ejemplo, las organizaciones gubernamentales); b) vínculos funcionales, los que se encuentran a la entrada (input) y salida (output) del sistema, como es el caso de los turistas, operadores turísticos, proveedores, distribuidores, etc; c) vínculos normativos, aquellos grupos que comparten valores similares con el sistema, guían, asesoran y apoyan la eficiencia del sistema (competidores, asociaciones empresariales y profesionales) y d) vínculos difusos, aquellos stakeholders cuya contribución no puede ser claramente definida, por ejemplo, los vínculos con los medios de comunicación, líderes de opinión o la comunidad). Ante estas perspectivas, tanto la Teoría de los Stakeholders como la Teoría de Vínculos es procedente su aplicación al sistema turístico de salud (Zermeño, 2017).

\subsection{Interlocución del Análisis de Redes Sociales (ARS)}

Ahora bien, el intercambio de información entre cada actor de turismo de salud puede analizarse a través de un modelo de interlocución denominado redes, las cuales son definidas como vínculos formales e informales entre proveedores, individuos, pacientes y facilitadores de la actividad. Los vínculos entre nodos o actores se fortalecen en vista de grados de confianza, mismos que para Luna y Velasco (2005), pueden sintetizarse en tres tipos básicos: la confianza personal (normativa), la estratégica (calculada) y la basada en las capacidades (prestigio). De esta forma, los actores pueden vincularse considerando la 
amistad, parentesco o comparten una serie de normas o valores comunes. Otros en cambio, se conectan porque saben que la cooperación les traerá beneficios. Así también, la relación se fundamentará en el respeto y prestigio de los participantes (tratándose de individuos u organizaciones) en términos de sus capacidades y competencias, tales como recursos, experiencia, conocimientos, confiablidad y compromiso.

Tales distinciones sobre la confianza, permite suponer que los vínculos reticulares entre actores y stakeholders de turismo de salud se fortalecen en vista de la confianza que se permea entre ellos, lo que favorece la colaboración en acciones de desarrollo del destino. El enfoque estructural de redes sociales ha sido retomado por diversas investigaciones de turismo (Adenekan, 2017; Baggio, Scott y Cooper, 2010) dado el carácter sistémico de los grupos interesados y la fortaleza de sus relaciones. Asimismo, han sido recientemente estudiadas en el ámbito de la salud, por ejemplo, en términos del manejo de pacientes (Ferreira et al., 2013), para comprender la toma de decisiones de los médicos (Cohen et al., 2013), o para comprender las razones de los viajes del turista de salud (Hanefeld et al., 2015).

Con objeto de exponer el enfoque de redes se expresa que los ARS utilizan normalmente dos aproximaciones para explicar los efectos limitantes o potenciadores y para interpretar su comportamiento social. Una aproximación es llamada "relacional" que se centra en las conexiones directas e indirectas entre actores. En esta aproximación, se pueden identificar conexiones fuertes, sólidas y confiables, pero también vínculos débiles que unen a actores de forma indirecta o redes aisladas dentro de la estructura (Granovetter, 1973, Rodríguez, 2005). Por lo tanto, en cuanto más fuertes son los vínculos entre los actores más similares son éstos. Los vínculos fuertes sugieren redes compactas, pero con información redundante en su interior. Mientras que en los lazos débiles conectan a los actores con otros elementos distantes permitiendo una mayor circulación y difusión de diferentes tipos de información.

En el ARS, como lo indica Scott (2017), los apegos individuales pasan a segundo término para priorizar los sistemas relacionales de los agentes constituidos a partir de los pares conectados, realizando análisis estadísticos del conteo de las relaciones, así como descripciones más globales sobre la estructura y el desarrollo de la red.

Dentro de la Teoría de Redes, es importante también, ponderar las posiciones de los nodos a través de medidas de centralidad (Molina, 2005; Freeman, 2012). Las mediciones centrales más comunes en la estructura de la red es el grado nodal, definido como el número de actores a los que un actor está directamente unido, y la dirección de las relaciones, que pueden ser de salida (las relaciones que un actor tiene con otros actores), y de entrada (el número de actores que tienen relaciones con él).

Otras medidas que fundamentan el grado de importancia de un actor recaen en su intermediación (betweenness) y en su cercanía (closenness). La primera, se enfoca en el control de comunicación, y se interpreta como la posibilidad que tiene un nodo o actor para intermediar las comunicaciones entre pares de nodos. Las medidas de intermediación de un nodo se obtiene al contar las veces en que este aparece en los caminos (geodésicos) que conectan a todos los pares de nodos de la red, a estos actores se les llama "actores puente". Por su parte, el grado de cercanía o closenness es la capacidad de un nodo de llegar a todos los actores de una red, esta se calcula al contar todas las distancias geodésicas de un actor para llegar a los demás (Velázquez y Aguilar, 2005; De Nooy et al., 2011). Así también, es indispensable la medición de la densidad total de la red, que permite medir la proporción de relaciones existentes sobre el total de relaciones posibles, indicando, por tanto, la intensidad de las relaciones en el conjunto de toda la red (Merinero, 2010). 
De esta manera, al utilizar la teoría de redes, es posible describir el comportamiento de relaciones de los distintos actores en su entorno, trátese de individuos, organizaciones o unidades sociales colectivas (Zizaldra, 2009) y la generación de acciones dirigidas a innovar en procesos, servicios, y gestión (Zermeño, 2017).

\subsection{Redes de colaboración para la innovación}

La cualificación de la innovación en los servicios en general y particularmente en los servicios turísticos resultan complejos por las formas en que se ofrecen al cliente y las formas en que se relacionan con otras empresas (por ejemplo, las alianzas estratégicas) o en la forma de transferencia del servicio. Por otra parte, Robayo (2016), indica que la innovación en los servicios suele ser flexible en su significado y no tiene que estar basada solamente en ideas novedosas, sino también, y más frecuentemente, se da mediante la implantación de pequeñas mejoras en productos o procesos.

Asimismo, la naturaleza de las actividades innovadoras varía considerablemente de una empresa a otra. Tal como lo menciona Pavitt (2003), la innovación es contingente y puede variar de organización a organización de acuerdo con el país o la industria en que se encuentre, el tamaño, la experiencia y la propia experiencia innovativa. Por ejemplo, en algunas organizaciones la innovación puede estar asociada a la introducción de un único cambio, mientras que en otras se generan innovaciones a través de cambios progresivos que finalmente impactan en un cambio significativo.

Además, en algunos sectores y organizaciones, los procesos de innovación se caracterizan por su informalidad, tal como lo describe Malaver y Vargas (2004), debido a que no se circunscriben a procesos planeados y por originarse en la solución de problemas como respuesta a las necesidades de los clientes o en el aprovechamiento de oportunidades que les dicta el mismo mercado (Zermeño, 2017).

Pero trabajar en estrategias que mejoren la experiencia del consumidor no es una tarea que corresponda solamente al prestador del servicio. Burns (2004), recomienda los sistemas que dan prioridad a los esfuerzos de colaboración que pueden unir instituciones y sistemas en lugar de operar aisladamente. Por lo tanto, hay una necesidad de integrar marcos que entiendan las múltiples interrelaciones entre los factores dispares, como turistas, operadores turísticos, propietarios de pequeñas empresas de turismo, miembros de la comunidad y Gobierno (Saxena, 2005).

En virtud de ello, se reconoce en el análisis de la literatura turística que el conocimiento y la adquisición de conocimiento a través de redes desempeña un papel vital para la innovación (Scott, Baggio y Cooper, 2008; Thomas y Wood, 2014). Es decir, las estrategias de innovación y las políticas en este sentido, se creen a partir de redes de colaboración entre las partes interesadas para desarrollar ideas de negocio (Nordin, 2003).

Por otra parte, en lo referente a la innovación en el turismo de salud existe una literatura limitada y sólo en los últimos años han aparecido investigaciones en este rubro. En este sentido, Szymanska (2015) señala que el modelo de Sistemas en Red es el más conveniente para tratar los procesos de innovación. Esto significa que las conexiones flexibles en la transferencia de conocimiento entre las organizaciones públicas y privadas, y un contacto permanente con el consumidor permitirá la aplicación de innovaciones en la actividad del turismo de salud. 


\section{Metodología}

La presente investigación es un estudio descriptivo de carácter cuanticualitativo a través del método de estudio de casos, llevada a cabo en la ciudad de Los Algodones, Baja California (LAL), elegida por sus ventajas geoestratégicas al localizarse en la zona noroeste de México, manteniendo una frontera geográfica y política con la población de Andrade, California en Los Estados Unidos. Así como ser identificado, desde hace más de 40 años, como un destino de turismo de salud, reconocido por turistas nacionales y extranjeros, provenientes principalmente de Estados Unidos y Canadá, que solicitan servicios médicos y de salud más frecuentemente en las temporadas invernales, por lo que se les ha dado en llamar snowbirds.

De igual manera, se eligen LAL, por contar con una infraestructura instalada, relativamente fuerte, de clínicas dentales, consultorios de medicina general y de especialidad, ópticas, spa, hoteles, farmacias y otros servicios turísticos; además de una coordinación de actores y stakeholders de turismo de salud a través de organizaciones que las representan para la búsqueda de la competitividad del destino.

El enfoque de trabajo consistió en la aplicación del método de ARS considerando que las redes son organismos sociales complejos, conformados por actores formales e informales, que sólo a través de entrevistas semiestructuradas a profundidad puede revelarse la dinámica de las relaciones entre ellos. Por consiguiente, se aplicaron 23 entrevistas semiestructuradas a actores y stakeholders de la comunidad de LAL que participan directa o indirectamente en el turismo de salud y que por su activismo en esta actividad económica se consideran líderes de opinión y colaboradores en la implementación de estrategias de innovación.

En el diseño de los instrumentos de recolección de información en el método de ARS, Rodríguez (2005) recomienda el uso de cuestionarios o entrevistas semiestructurada que permita la nominación de actores, haciendo uso de preguntas acerca de sus relaciones personales, lazos familiares, de amistad, agrupaciones de trabajo, etc. En consideración a lo anterior, el uso de preguntas incluyó nombrar colegas del sistema turístico con los que se reúne a eventos familiares, profesionales, sociales o comunitarios y con los que se agrupa para generar iniciativas de innovación sobre el destino; así como una conversación abierta sobre los tipos de innovación por colaboración en el orden de producto, proceso, organización y mercadotecnia. Dicha información fue grabada (previa consulta de los participantes) y transcrita posteriormente para efectos de analizar su contenido. Todo el proceso de recolección de la información se desarrolló durante el periodo de octubre del 2016 a abril del 2017.

El método de muestreo implementado fue la técnica de "bola de nieve", iniciando con la identificación de los participantes líderes de opinión pertenecientes a Prestadores de servicios de salud; Gobierno Delegacional de Algodones; Comité de Desarrollo Turístico de Algodones, A. C.; Consejo de Turismo Médico de Algodones; Prestadores de servicios turísticos; y Comité Cultural de Algodones. Los cuales configuran:

- $\mathrm{AE}=$ Asociación empresarial

- $\quad$ EPSS = Empleado de servicios de salud

- $\mathrm{G}=$ Organización gubernamental

- PSS = Prestador de servicios de salud

- PST = Prestador de servicios turísticos 
- Revista de Ciencias Sociales y Humanidades. ISSN-P: 0188-9834 ISSN-E:2395-8669.

Una vez encuestados por medio de entrevistas semiestructuradas se decide terminar el proceso de "bola de nieve" cuando se ha nombrado, repetidamente, a los líderes más significativos de turismo de salud en la región de LAL. En este entendido, el proceso puede sintetizarse en el siguiente:

a). Identificación de los actores principales de cada grupo de interés para iniciar el muestreo "por bola de nieve".

b). Elaboración de matriz de relaciones cuadrática, denominada matriz adyacente, formada por columnas (como si fuesen variables) y a su vez en filas (como si fueran casos).

c). Para la conformación de la estructura reticular de los actores se alimenta con la matriz adyacente el software informático profesional para el ARS: PAJEK, como herramienta para el análisis y visualización de redes de colaboración, a través de la implementación de una serie de algoritmos matemáticos (De Nooy et al, 2011).

d). Obtención de la red de relaciones en el sistema informático, donde los actores son diferenciados con figuras geométricas. Con esta red construida se efectúan diferentes mediciones:

i. Cálculo de la densidad de la red.

ii. Cálculo de la centralidad de entrada, salida y total.

iii. Cálculo de la centralidad de intermediación (betweenness).

iv. Cálculo de la centralidad de cercanía (closeness).

d). Las redes y la cuantificación de centralidad se interpretan en relación a los supuestos planteados y se generan conclusiones.

\section{Resultados}

Como puede observarse en la Figura 1, la Teoría de los Stakeholders como la Teoría de Vínculos es procedente su aplicación al medio turístico y salud. La interlocución de público, privado como de la academia y de organismos no gubernamentales. De tal manera, que el interés de valorar la reticularidad de actores y stakeholders de turismo de salud en el área de influencia de LAL se llevó a cabo encuesta por medio de entrevistas semiestructuradas, información analizada bajo la perspectiva de ARS. 
Figura 1. Relaciones entre actores y stakeholders de turismo de salud

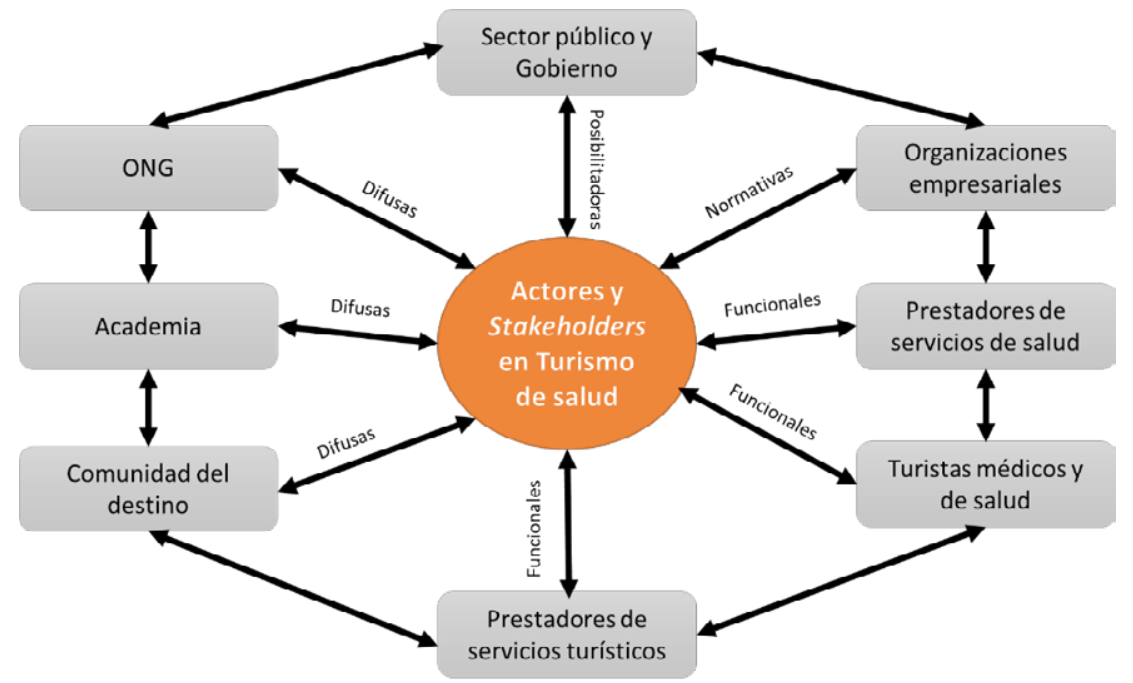

Fuente: Elaboración propia con base en la Teoría de Vínculos (Gruning y Hunt, 1984).

La encuesta desarrollada permitió la generación de relaciones entre los diferentes participantes, denominados en forma genérica por el modelo de ARS, como "actor" y a la relación entre ellos se le denomina vínculo e implica el flujo de información entre los integrantes del sistema de turismo de salud.

Con apoyo del Sistema informático PAJEK se construyeron las estructuras reticulares que muestran las relaciones de amistad (véase en la Figura 2); de confianza (véase en la Figura 2; respeto (véase en Figura 3) y colaboración para la innovación (véase en Figura 4). Lo anterior, justificado en el supuesto de que la red de actores se encuentra afianzada en las relaciones de amistad, confianza, respeto y compromiso al desarrollo de la actividad de turismo de salud.

Asimismo, se presentan los resultados del cálculo de la densidad de las redes para identificar la fortaleza de la red o redes encontradas; así como el cálculo de medidas de centralidad: de entrada, salida y total; centralidad de intermediación (betweennes) y centralidad de cercanía de los actores (closeness).

Prosiguiedo con el análisis, se presenta la Figura 2, que muestra la estructura de las relaciones entre actores que se perciben con mayor de confianza en el trabajo profesional, tanto dentro como fuera de sus organizaciones de origen, en evidencia de que las redes basadas en la confianza permiten el desarrollo de la colaboración y la innovación. Las figuras en triángulos corresponden a las generaciones con una antigüedad en el sistema turístico de Los Algodones de menos de 20 años. Los círculos, corresponden a los actores con una antigüedad de más de 20 años en el sistema, considerando que el tiempo de residencia en el sistema turístico de salud genera una mayor compromiso a la colaboración en beneficio del desarrollo del destino. Esta nomenclatura en las figuras se repetirá a lo largo de todas las redes expuestas. Asimismo, el tamaño de los actores corresponde a la centralidad total o grado nodal mayor; es decir, entre mayor dimensión tenga la figura, mayor serán sus vínculos de entrada y de salida. 
Figura 2. Red de confianza entre actores y stakeholders de turismo de salud en la ciudad de Los Algodones

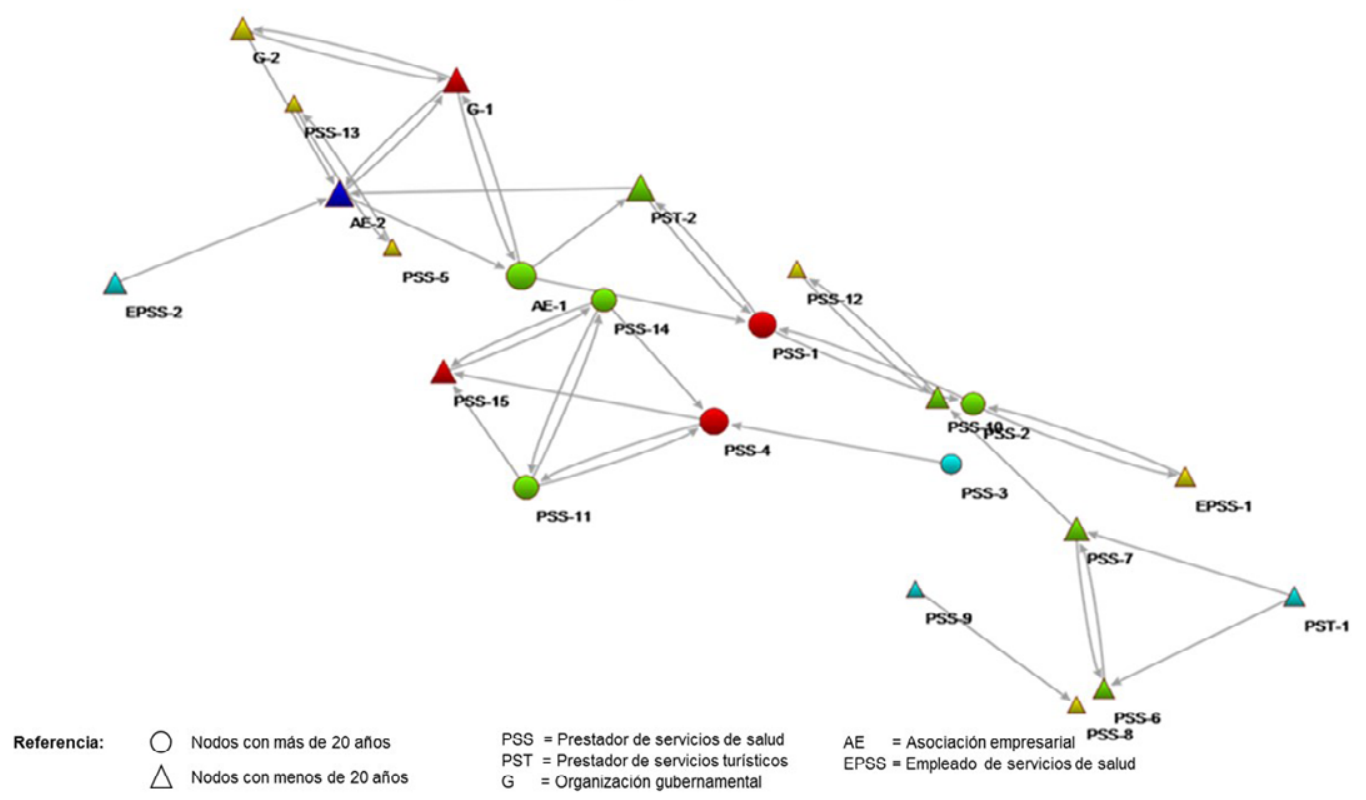

Fuente: Elaboración propia con base a PAJEK

En base a la estructura de la red anterior, se muestran las medidas más significativas para valorar la reticularidad de actores y stakeholders. Primeramente, se calcula la densidad de la red, que permite identificar la relación entre los vínculos presentes en la misma y el número de vínculos posibles multiplicado por 100. La red de confianza posee 38 vínculos y 23 actores encuestados en esta sección. De esta forma, el número de vínculos posibles es igual al número de nodos (actores) multiplicado por el número de nodos menos 1; es decir, para este caso, el número de vínculos posibles son 506 y el número de vínculos de la red son 38. En consecuencia, la densidad de la red es de $7.5 \%$.

A continuación, en la Figura 3, se presenta la estructura reticular de los actores y stakeholders por los que se muestra mayor respeto en el sistema de turismo de salud por su trabajo profesional, mostrando evidencia de liderazgo en las iniciativas de colaboración para el desarrollo de la actividad turística del destino. De modo que los actores a quienes se reconoce con mayor respecto se observan en las figuras de mayor tamaño. Con respecto a la densidad de la relación entre los 23 actores encuestados es de 6.3\%. 
- Revista de Ciencias Sociales y Humanidades. ISSN-P: 0188-9834 ISSN-E:2395-8669.

Figura 3. Red de respeto entre actores y stakeholders de turismo de salud en la ciudad de Los Algodones

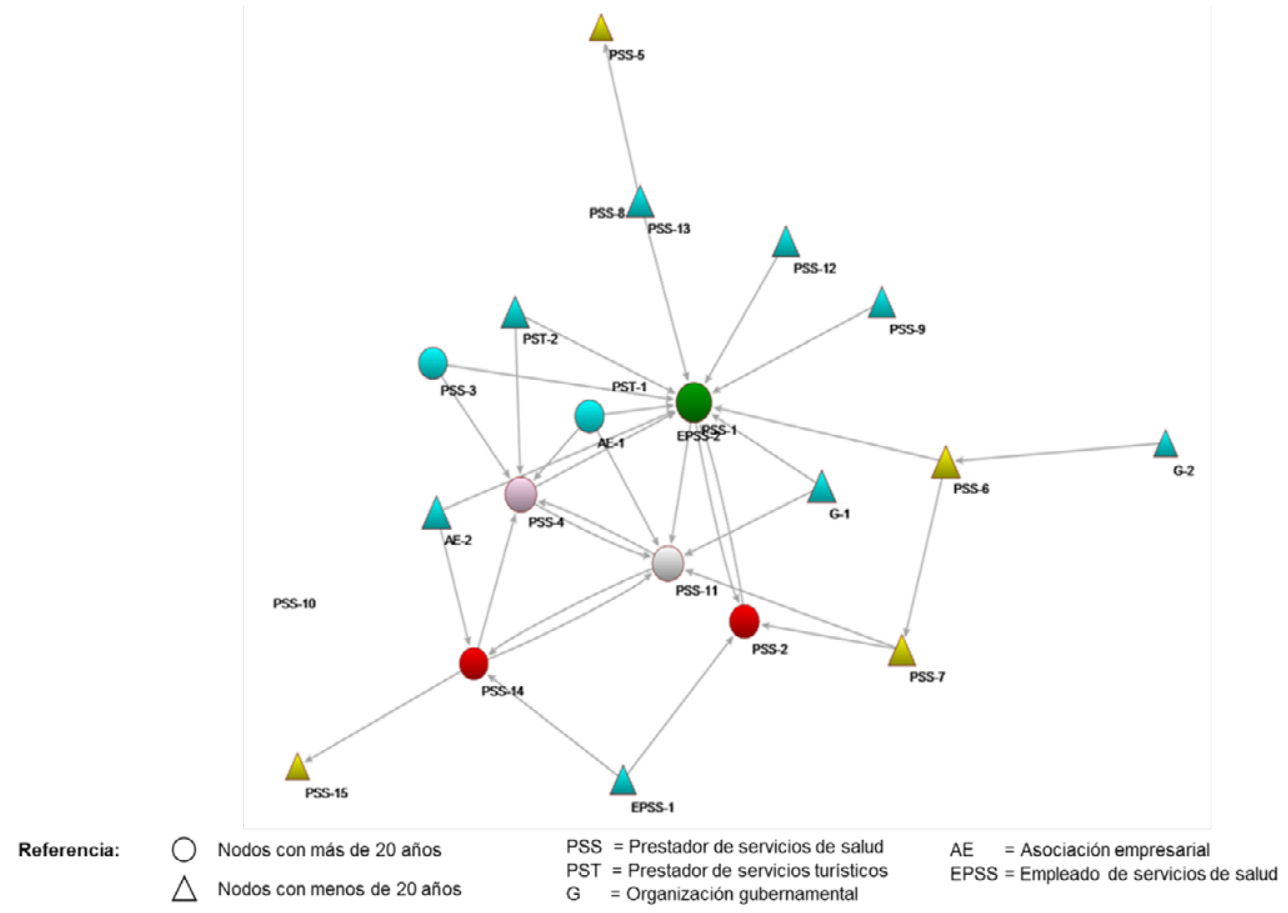

Fuente: Elaboración propia con base a PAJEK

Por otra parte, en Figura 4, se presenta la estructura reticular de los actores y stakeholders que reconocen la colaboración con otros actores para plantear procesos de innovación en el turismo de salud, siendo a su vez promotores del destino. En consideración a lo anterior, la densidad de la relación entre los 23 actores encuestados es de $10 \%$. 
Figura 4. Red de colaboración entre actores y stakeholders para la innovación en turismo de salud en la ciudad de los Algodones

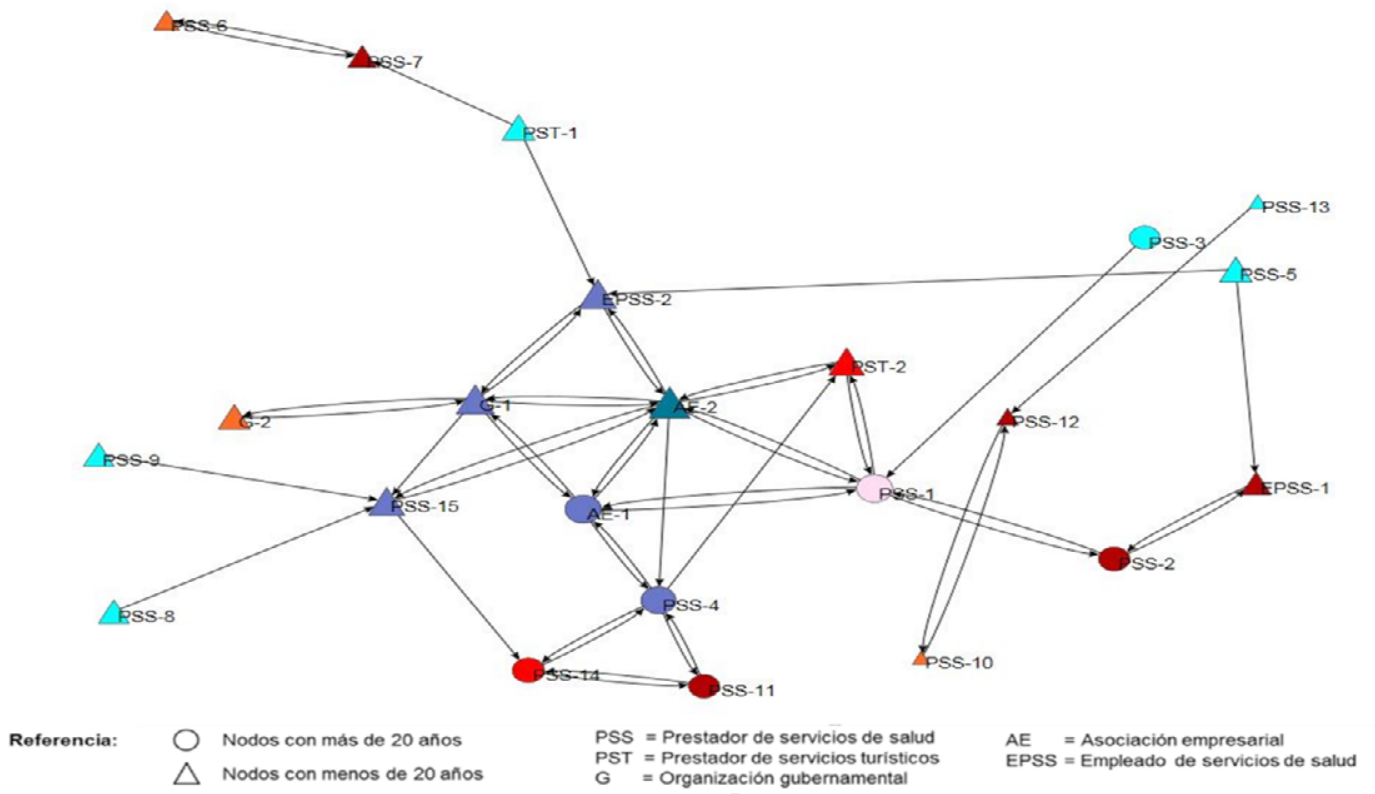

Fuente: Elaboración propia con base a PAJEK

Recapitulando, los resultados presentados en las figuras, muestran tres aspectos reveladores: la estructura reticular entre actores y stakeholders sobre turismo de salud en la región de influencia de Los Algodones; la condición relacional de confianza, respeto y colaboración que facilita el proceso de innovación para el desarrollo de la actividad turística y salud en la región.

\subsection{Discusión de resultados}

La constitución del trabajo cooperativo entre las organizaciones de turismo de salud del destino de LAL se presenta como una solución bastante promisoria, pasando de la competencia pura a acciones de desarrollo conjunto como la conformación del Consejo de Desarrollo Turístico de Algodones, A. C. (COTUDEAL) y el Consejo de Turismo Médico, ambas bajo los esfuerzos de la iniciativa privada.

Así, se evidencia que las redes entre actores y stakeholders de turismo de salud se encuentran afianzadas en relaciones de confianza, respeto y compromiso en el desarrollo de la actividad turística de la región. Lo anterior, se refleja en el comportamiento reticular de la agrupación de actores en diferentes acciones de la vida en comunidad dentro de la actividad de turismo de salud. Se rescata el uso de las redes sociales, donde una red de individuos con líneas de confianza puede proveer medios para reconocer oportunidades, establecer estrategias conjuntas de desarrollo, promover la innovación abierta y ser potencialmente fuertes en ventajas competitivas. Si bien es cierto, existe vaga evidencia de la naturaleza de las fuerzas motrices en los sistemas de innovación, si puede indicarse lo que al tema indica Nordin (2003) y Hjalager (2010), que las relaciones humanas y las estructuras interorganizacionales se consideran par- 
ticularmente importantes en los procesos de innovación, así como la colaboración abierta, convincente e intersectorial explica parte del éxito de los destinos turísticos.

De esta forma, las redes de confianza de LAL facilitan en gran medida la interacción entre actores, agrupando los contactos con intereses comunes, permitiendo el flujo de información y la generación de procesos creativos. De acuerdo con el estudio, la estructura reticular muestra una densidad de $7.5 \%$ con respecto a la esperada de $13.6 \%$, en consideración a que cada actor pudo nombrar a tres actores como máximo. Dado lo anterior, en la Figura 2, sobresale la centralidad normalizada de los nodos AE-2 (13\%) y el G1 (13\%), distribuyéndose el poder hegemónico en toda la red. Es decir, la estructura de la red es de tipo policéntrica. En cuanto a los valores de intermediación mayor se destaca el PSS-2 con un valor de 0.28139 , fungiendo como el actor que permite conectar al $28 \%$ de los miembros de la red. Igualmente, destaca el actor AE-1 como el que con mayor rapidez se conecta con el resto de los miembros; así, su valor de cercanía normalizada es de $24 \%$. Lo anterior, es significativo al ser el Presidente del Consejo de Desarrollo Turístico de Algodones, A. C. (COTUDEAL, A. C.).

Con la intención de iniciar el proceso para determinar los nodos más condensados y fuertes de la red, se estructuran las relaciones en base al respeto que actores y stakeholders muestran ante sus colegas y sobre los que decanta su bienestar personal y en los que confiarán para las decisiones sobre el manejo de la actividad turística. Sin embargo, se identifica una densidad de $6.3 \%$ en comparación a la máxima esperada de $13.6 \%$, debido a que los actores nominados pertenecen sólo al grupo de Prestadores de Servicios de Salud (PSS), como puede observarse en la figura 4 por los nodos sueltos y los ubicados en la periferia de la red. Por otro lado, destaca la figura policéntrica de la red en virtud de nodos centrales como el actor PSS-1, PSS-4, PSS-14 y PSS-11, pioneros en la prestación de servicios de salud en la región con más de 20 años apostados en la ciudad de LAL. Asimismo, se enfatiza la posición de respeto y activismo en el desarrollo de la actividad de turismo de salud del actor PSS-1, con la centralidad mayor (27\%); el grado más alto de intermediación (7.7\%) y manteniéndose como el actor más cercano con un nivel de 59.5\%. Es decir, se posiciona como el líder en el reconocimiento público que colegas y comunidad le otorgan en su actividad profesional.

Con respecto a las relaciones de colaboración para generar procesos de innovación, se presenta la Figura 4, como una red central condensada con una densidad de 10\% en comparación con la máxima esperada de $13.6 \%$. No obstante, se identifican actores que mantienen una baja coordinación con otros actores encuestados para el desarrollo conjunto de iniciativas de innovación. El actor AE-2 se exhibe como un promotor en la coordinación de acciones de innovación, situación que se justifica al ser parte del Consejo de Desarrollo Turístico de Algodones (COTUDEAL). Así también, el actor AE-2 muestra la centralidad normalizada mayor del $29 \%$; una intermediación de $9.7 \%$ y una cercanía de $37.7 \%$. A este respecto, Hjalager (2010) observa que, debido a las redes sociales y a su proximidad, las innovaciones son rápidamente difundidas e implementadas.

A lo largo de las diferentes estructuras que demostraron la reticularidad de actores y stakeholders de turismo de salud, se observaron nodos fuertes y condensados que cooperan con la actividad del turismo de salud en el destino, así como apoyo a la comunidad. El perfil de los nodos que componen esta red es de agrupaciones con más de 20 años laborando en la zona, que viven en la región de influencia (Los Algodones, San Luis Río Colorado). Asimismo, su grado de profesionalización es mayor y se concentran en el primer cuadro de la ciudad. Por otra parte, los actores de agrupaciones de menos de 20 años de 
experiencia, se asemejan a redes de nodos débiles, donde los niveles de cooperación son menores y la colaboración en procesos de innovación suele ser limitada al interior de la red. Así pues, como señala Hjalager (2010), las redes sociales relativamente débiles sugieren una inclinación limitada a aprender de los demás y participar en la colaboración, tanto a nivel local como más ampliamente. Por lo tanto, se recomienda que los esfuerzos de colaboración se detenten entre actores homogéneos en el nivel de destino, permitiendo avanzar en procesos de innovación más planeados.

Para Granovetter (1973), en los lazos débiles existe poco compromiso, afecto y confianza entre los actores. No obstante, los nodos débiles permiten el acceso a nueva información. Afirma que cuanto más fuertes son los lazos más similares son los actores que los componen con información redundante en su interior, en cambio, los lazos débiles manejan información más novedosa constituyendo la principal fuente de innovación de la organización (Zermeño, 2017).

Por consiguiente, la colaboración permite generar propuestas de mejoras en el sistema de organizaciones de turismo de salud. La fuerza de la red genera una sinergia para promover la creatividad e innovación tanto en los productos y servicios, los procedimientos, la organización y la mercadotecnia. En el sistema turístico de LAL sobresalen las estrategias de innovación en el ámbito no tecnológico; es decir, en la organización y en mercadotecnia; mostrándose con la introducción de cambios de magnitud diversa y de bajo grado de novedad en comparación con las innovaciones introducidas por empresas industriales o en grandes corporaciones, pues como lo indica Lynch y Morrison (2007), la colaboración y las redes para la innovación suelen ser preponderantes en empresas grandes. Sin embargo, las organizaciones turísticas en el destino de LAL han permanecido como sistemas informales de gestión por lo que resulta altamente significativo los esfuerzos de imitación creativa, al incorporar cambios en vista del éxito en otras organizaciones del área y en el esfuerzo conjunto en el fomento de la calidad y promoción del turismo de salud fundamentado en la colaboración entre los prestadores de servicio de salud, prestadores de servicios turísticos, asociaciones empresariales y gobierno.

En los comentarios vertidos en las entrevistas semiestructuradas se pone de relieve que a pesar que la innovación se genera al interior de los negocios, el fomento a la calidad y promoción del turismo de salud es un trabajo conjunto fundamentada en la colaboración entre los distintos grupos de interés. A este respecto, indican que la innovación en los servicios (de producto) puede observarse en la instrumentación de servicios adicionales a los de práctica médica. Por ejemplo, el ofrecer paquetes completos que incluyan el servicio médico, la transportación, el hospedaje, medicamentos y otros servicios y/o productos de cortesía. Otras acciones corresponden a la apertura de nuevas líneas de negocios de servicios turísticos y de salud como; clínicas dentales, ópticas, clínicas de células madres, hoteles, servicios de cámaras hiperbáricas, spa, restaurante, tiendas de artesanías, joyerías, farmacia, entre otros (Zermeño. 2017).

En relación con innovaciones de proceso, se evidencian en la estandarización de los tiempos de atención, implementando sistemas de información para control del turista de salud, instrumentación de protocolos médicos. Por su parte, la innovación en la organización puede observarse en la incorporación de herramientas administrativas como análisis FODAS, cadenas de valor, modificaciones a la estructura jerárquica de los negocios y la toma de decisiones.

Las innovaciones en el área de mercadotecnia son diversas en el medio interno de las organizaciones prestadoras de servicios para el fomento del turismo de salud en la región. Algunos prestadores de servicios médicos y otras empresas como hoteles y restaurantes compran espacios de publicidad en las ciudades 
de Yuma y Phoenix en Arizona y otros en el Centro, Calexico y San Diego en el Estado de California en los Estados Unidos. Asimismo, de manera conjunta, participan con actividades artísticas y sociales llevadas a cabo en la zona, con la cooperación económica y humana de la mayoría de los establecimientos asentados en el lugar. Son ejemplos, la fiesta de bienvenida de "Los Pájaros de la Nieve", la festividad de Día de Muertos, entre otros. Es esta colaboración que permite generar propuestas de mejoras al interior de las organizaciones de salud, en otras palabras, la fuerza de la red genera una sinergia para promover la creatividad e innovación tanto en los productos y servicios, los procedimientos, la organización y la mercadotecnia.

\section{Conclusión}

Los actores turísticos se enlazan en redes por distintas razones y para diversos objetivos. No obstante, la fortaleza de los lazos las crean los individuos y las organizaciones en la medida que confían en el otro, respetan su capacidad para tomar decisiones, dado el compromiso que perciben de estos para desarrollar la actividad del turismo.

No obstante, una persona u organización puede tener un prestigio firme, respaldado por sus competencias y capacidades, y no necesariamente, ser reconocido como un actor fiable para la toma de decisiones. En estos casos, se valoran los lazos fuertemente vinculados por la combinación de una confianza personal, normativa y técnica. De acuerdo a lo anterior, los actores más centrales en la red, en sus diferentes dimensiones (amistad, confianza, respeto, colaboración), suelen ser factores importantes en la construcción de liderazgo dentro de la red.

Asimismo, se trata de vínculos densos y cerrados, caracterizados por un alto grado de interconexión entre los nodos, donde los individuos colaboran porque comparten un conjunto de valores y normas. También se trata de individuos con atributos semejantes (valores, cultura, educación, asentamiento en el lugar, provenientes de similares instituciones de educación superior). Por lo tanto, suelen ser (formal e informalmente) actores que encabezarán las decisiones de cambios a largo plazo en el destino. En el caso particular de LAL; los Prestadores de Servicios de salud PSS-1, PSS-4, PSS-15 y el representante de la Asociación Empresarial AE-2; se les atribuye el liderazgo en la iniciativa de encabezar proyectos de innovación. De esta forma, queda evidenciado lo que indica Gruning y Hunt (1984) en su Teoría de Vínculos en los stakeholders, donde los lazos funcionales, en este caso representados por los Prestadores de Servicios de Salud (PSS), encabezan el sistema turístico con proyectos, innovaciones y acciones para desarrollar el destino y fortalecer al sistema turístico. A su vez, los vínculos normativos, representados por las Asociaciones Empresariales (AE), guían y asesoran al sistema al logro de los objetivos planteados.

Se puede afirmar que las redes entre actores y stakeholders de turismo de salud se encuentran afianzadas en relaciones de amistad, confianza, respeto y compromiso al desarrollo de la actividad turística de la región de Los Algodones. Situación que se evidencia dada la estructura reticular de actores y stakeholders, fundamentadas en la mutua confianza, respeto y colaboración de sus pares de trabajo.

Las redes en Los Algodones muestran medidas de dispersión del 67\% en promedio. En términos turísticos, esto significa que las partes interesadas manifiestan un grado de colaboración a nivel de formación, una medida cuantitativa que se deriva de las métricas usadas por el análisis de redes (Freeman, 1979), donde, el coeficiente de agrupamiento (medio en este caso), puede ser considerado como representación 
a la tendencia a formar grupos colaborativos. Estos indicios de colaboración entre actores y stakeholders ha favorecido la capacidad de innovación abierta al reproducir acciones que favorecen la calidad de los servicios de una organización a otra. Sin embargo, las iniciativas son todavía poco planeadas y sin medidas estratégicas a largo plazo.

Además, tanto el número, como la composición de conglomerados identificados, exhiben propiedades de auto-regulación que conduce a la formación, en cierta medida, de una aglomeración de lazos y produce una serie de comunidades informales y una estructura comunitaria informal basada en las relaciones de afinidad personal, familiar, de amistad, respeto y reconocimiento público de los actores participantes.

Por otra parte, es positivo encontrar en la conformación de la estructura de las redes de colaboración, la participación de subgrupos diversos, identificándose redes de tipo policéntricas con un grado bien identificado de cohesión local. Ello respalda la idea de que los actores y stakeholders de la actividad turística deben ser alentadas a cooperar para intercambiar conocimientos y, por tanto, aumentar la competitividad del destino.

En vista de los comentarios vertidos por actores y stakeholders en las entrevistas semiestructuradas, se concluye que la capacidad de innovar no es sólo una destreza individual; sino más bien competencia social compartida por los actores sociales que forman parte del sistema económico de turismo de salud. Lo anterior, también lo reafirman Burns (2004), Carlise et al (2013) y Robayo (2016), al indicar que el proceso generador de ideas (creatividad) y su implementación (innovación) es un proceso colectivo que se ha convertido en una fuente de ventaja competitiva.

En el caso del fenómeno estudiado, el proceso de innovación se identifica de carácter informal y no sistematizado, ya que busca, como lo señala Robayo (2016), el aprovechamiento de oportunidades del mercado y, por lo general, provienen de la exigencia y de las necesidades de los clientes, o como reacción a las circunstancias inducidas por la competencia, pero carecen de una planificación a largo plazo.

Se indica asimismo, la necesidad de una mayor profundización en el análisis de las estructuras entre actores y stakeholders en turismo de salud, la fortaleza de sus lazos, los subgrupos o cliques dentro de la estructura, y los resultados obtenidos por la colaboración entre los diferentes actores, información que permee de nueva luz sobre los mecanismos de agrupación y las iniciativas de innovación que fluyen entre los vínculos de la red. Por otro lado, el ARS como una técnica de análisis estructural, es sin duda una alternativa metodológica en el estudio del turismo, reconociendo la naturaleza relacional de gran parte de sus actividades económicas.

\section{Referencias}

Adenekan, Nick. 2017. Creating sustainable tourism ventures in protected areas: An actor/network theory analysis. Tourism management, 61: 161-172. DOI: https://doi.org/10.1016/j.tourman.2017.02.006 Antón, Salvador y Juan Duro. 2009. Competitividad y sistemas de innovación territorial en turismo. INNOVA, 1-37.

Baggio, Rodolfo. 2008. Network analysis of a tourism destination. Tesis de doctorado. Australia: University of Queensland. 
Baggio, Rodolfo, Noel Scott y Chris Cooper. 2010. Network science. A review focused on tourism. Annals of Tourism Research, 37(3), 802-827. DOI: https://doi.org/10.1016/j.annals.2010.02.008

Baro, Marcelo. 2011. Jerarquización de stakeholders para la construcción del capital social de las organizaciones. Mediaciones Sociales, 9: 135-162.

Burns, Peter. 2004. Tourism planning: a third way? Annals of Tourism Research, 31(1): 24-43. DOI: https://doi.org/10.1016/j.annals.2003.08.001

Carlise, Sheena, Martin Kunc, Eleri Jones y Scott Tiffin. 2013. Supporting innovation for tourism development through multi-stakeholder approaches: Experiences from Africa. Tourism Management, 35: 59-69. DOI: https://doi.org/10.1016/j.tourman.2012.05.010

Cohen, Dikla, Meira Levy, Orit Cohen y Khaled Karkabi. 2013. The influence of a professional physician network on clinical decision making. Patient Educational Cons, 93, 496-503.

Decelle, Xavier. 2004. A conceptual and dynamic approach to innovation in tourism, OCDE, 1-16.

De Nooy, Wouter, Andrej Mrvar y Vladimir Batagelj. 2011. Exploratory social network analysis with Pajek. Nueva York, USA: Cambridge University Press.

Ferreira, Adel, Taryn Young, Catherine Mathews, Moleen Zunza y Nicola Low. 2013. Strategies for partner notification for sexually transmitted infections, including HIV. Cochrane Data-base System. Rev, 10. DOI: http://doi.org/10.1002/14651858.CD002843.pub2

Freeman, Linton. 2012. El desarrollo del análisis de las redes sociales. Estados Unidos: Palibrio.

Freeman, Linton. 1979. Centrality in social networks conceptual clarification. Social Network, 1:215-239. DOI: https://doi.org/10.1016/0378-8733(78)90021-7

Freeman, Edward, Andrew Wicks y Bidhan Parmar. 2004. Stakeholders theory and the corporate objective revisited. Organizational Science, 15(3): 364-369. DOI: https://doi.org/10.1287/orsc.1040.0066

González, Rodrigo y Martín Mendieta. 2009. Reflexiones sobre la conceptualización de la competitividad de destinos turísticos. Cuadernos de Turismo, 23: 111-128.

Granovetter, Mark. 1973. The strength of weak ties. American Journal of Sociology, 78(6): 1360-1380. DOI: https://doi.org/10.1086/225469

Gruning, James y Todd Hunt. 1984. Dirección de las relaciones públicas. Barcelona: Gestión 2000.

Hanefeld, Johanna, Niel Lunt, Richard Smith y Daniel Horsfall. 2015. Why do medical tourism travel to where they do? The role of networks in determining medical travel. Social Science \& Medicine, 124: 356363. DOI: http://doi.org/10.1016/j.socscimed.2014.05.016

Hjalager, Anne. 2010. A review of innovation research in tourism. Tourism Management, 31, 1-12. https:// doi.org/10.1016/j.tourman.2009.08.012

Ivars, Josep, Fernando Vera y Alejandro Aceval. 2014. Políticas de innovación en turismo y desarrollo de clusters: la percepción gerencial en el programa Agrupaciones Empresariales Innovadoras (AEIs). Cuadernos de Turismo, 33: 97-120.

Luna, Matilde y José Velasco. 2005. Confianza y desempeño en las redes sociales. Revista Mexicana de Sociología, 67(1): 127-162.

Lynch, Paul y Alison Morrison. 2007. The role of networks. En Micro-Clusters and Networks. The growth of tourism. Micro- clusters and networks, editado por Ewen Michael. Oxford: Elsevier, 43-62. DOI: https:// doi.org/10.1016/B978-0-08-045096-4.50009-3 
Malaver, Florentino y Marisela Vargas. 2004. Los procesos de innovación en la industria colombiana: resultados de un estudio de casos. Cuadernos de Administración, 17(28): 9-51.

Merinero-Rodríguez, Rafael. 2010. Desarrollo local y análisis de redes sociales: el valor de las relaciones como factor del desarrollo socioeconómico. REDES-Revista Hispana para el Análisis de Redes Sociales, 18(11): 277-304.

Merinero-Rodríguez, Rafael y Juan Pulido-Fernández, J. 2016. Analysing relationships in tourism: A review. Tourism Management, 54: 122-135. DOI: https://doi.org/10.1016/j.tourman.2015.10.010

Molina, José 2005. El estudio de las redes personales: contribuciones, métodos y perspectivas. Revista de Metodología de Ciencias Sociales, 10: 71-105.

Molina, Sergio, Tomás Cuevas-Contreras e Ismael Rodríguez-Herrera. 2014. Desarrollo socioeconómico entre turismo y redes. En Complejidad del turismo en la frontera, editado por Alejandro Palafox y Tomás Cuevas. México. Editorial Gasca, 3-18.

Nordin, Sara. 2003. Tourism clustering and innovation. Östersund: ETOUR.

Pavitt, Keith. 2003. The process of innovation. Bringhton: University of Sussex.

Robayo, Paula. 2016. Revisión de la innovación como proceso y su gestión en la organización: una aplicación para el sector gráfico Colombiano. Suma de Negocios, 7: 125-140. DOI: https://doi.org/10.1016/j. sumneg.2016.02.007

Rodríguez, Josep. 2005. Análisis estructural y de redes. España: Centro de Investigaciones Sociológicas. Sautter, Elise y Birgit Leisen. 2009. Managing stakeholders: A tourism planning model. Annals of Tourism Research, 26(2): 312-328. DOI: https://doi.org/10.1016/S0160-7383(98)00097-8

Saxena, Gunjan. 2005. Relationships, networks and the learning regions: case evidence from the Peak District National Park. Tourism Management, 26(2): 277-289. DOI: http://doi.org/10.1016/j.tourman.2003.11.013

Scott, John. 2017. Social network analysis. Estados Unidos: SAGE.

Scott, Noel, Rodolfo Baggio y Chris Cooper. 2008. Network analysis and tourism: From theory to practice. Clevedon, UK: Channel View.

Szymanska, Elżbieta. 2015. Construction of the model of health tourism innovativeness. Procedia. Social and Behavioral Sciences, 213: 1008-1014. DOI: https://doi.org/10.1016/j.sbspro.2015.11.518

Thomas, Rhodri y Emma Wood. 2014. Innovation in tourism: Re-conceptualising and measuring the absorptive capacity of the hotel sector. Tourism Management, 45; 39-48. DOI: http://dx.doi.org/10.1016/j. tourman.2014.03.012

Vargas-Hernández, José Guadalupe. 2011. Intercambio transfronterizo de servicios de salud y medicinas en la región de Tijuana y San Diego. Revista de Pueblos y Fronteras Digital, 6(10), pp. 202-337.

Velázquez, Alejandro y Norman Aguilar. 2005. Manual introductorio al análisis de redes sociales. México: Ucinet.

Verschoore, Jorge y Alsones Balestrin. 2008. Factores relevantes para el establecimiento de redes de cooperación entre empresas de Rio Grando do Sul. RAC-Revista de Administración Contemporánea, 12(4): 1043-1069. DOI: http://dx.doi.org/10.1590/S1415-65552008000400008

Vilaseca, Jordi, Joan Torrent, Josep Lladós y Lluís Garay. 2007. Tecnologías de la información y comunicación, innovación y actividad turística: hacia la empresa en red. Cuadernos de Turismo, 19, 217-240. 
- Revista de Ciencias Sociales y Humanidades. ISSN-P: 0188-9834 ISSN-E:2395-8669.

Zermeño-Flores, Sonia. 2017. Gestión del capital intelectual e innovación en turismo de salud en la región de influencia de Vicente Guerrero (Los Algodones), Baja California. Tesis de doctorado. Ciudad Juárez: Universidad Autónoma de Ciudad Juárez.

Zizaldra, Isabel. 2009. Redes transfronterizas en turismo. Tesis doctoral. Universitat de Girona. Girona, España 